\title{
əOn the Nonlinear, Three-Dimensional Structure of Equatorial Oceanic Flows
}

\author{
A. CONSTANTIN \\ Faculty of Mathematics, University of Vienna, Vienna, Austria \\ R. S. JOHNSON \\ School of Mathematics, Statistics and Physics, Newcastle University, Newcastle upon Tyne, United Kingdom
}

(Manuscript received 31 March 2019, in final form 28 May 2019)

\begin{abstract}
A systematic development, based on the construction of an asymptotic solution of the Euler equation, written in rotating, spherical coordinates $(\varphi, \theta, r)$, is used to investigate the flows of the type seen in the neighborhood of the Pacific equator. First, it is shown that the observed poleward surface-flow structure away from the line of the equator is possible only if the flow evolves (changes) in the azimuthal direction. Then, allowing for variations in the azimuthal direction, the shallow-water, small-Rossby-number version of the problem, approximated close to the equator, leads to an asymptotic formulation that admits any prescribed azimuthal velocity profile $u(\theta, r)$ at some fixed longitude $\varphi$. The maximum extent of the flow region inside which we can describe in detail the velocity field is restricted by the size of the Rossby number. The analysis demonstrates that the meridional $v$ and vertical $w$ velocity components are nonlinearly connected to $u$, and that all three velocity components appear at the same order in the leading (scaled) equations, even though the physical size of $w$ is very much smaller than that of the other two components. An appropriate choice is made for $u$, at a given $\varphi$, and the corresponding complete threedimensional flow field, which emerges from the interlinkage of the velocity components, is described; the thermocline is also added to the flow configuration. We compare these results with the available field data, demonstrating that this formulation captures all the main structures of the flow field, but also allows for many choices to be made that can be used to adjust the details of the flow and to model other, similar flows.
\end{abstract}

\section{Introduction}

The ocean in the neighborhood of the Pacific equator possesses some intriguing properties. There is the surface current that flows to the west, in the neighborhood of the equator, and a stronger current below this, which flows to the east-the Equatorial Undercurrent (EUC), sometimes called the Cromwell Current (described by him and his coworkers in 1954) - while below the EUC there is a rapid decay of the flow field towards a practically still abyssal layer (see Boyd 2018; Gill 1982; Talley et al. 2011). In addition there is the presence of a pycnocline/thermocline that sits, approximately, at the level of maximum speed in the undercurrent. This persistent and coherent structure within the ocean exists

¿ Denotes content that is immediately available upon publication as open access.

Corresponding author: A. Constantin, adrian.constantin@ univie.ac.at in a band of about 300-km width across the line of the equator, and extends down to about $200 \mathrm{~m}$ or so; see Johnson et al. (2001) for a detailed description of this flow, obtained from shipboard observations. Although the general flow structure is well understood and fairly readily explained, there is no comprehensive and allembracing theory for this type of flow and which, for example, links all three velocity components in one simple formulation. Our aim is to present such a theory, based on the Euler equation written in rotating, spherical coordinates with the shallow-water approximation imposed and valid near the equator. The emphasis will be on showing how the three velocity components are intimately connected and that the profile in the azimuthal direction (this being the natural choice, on both mathematical and physical grounds) completely determines the other two.

This article is licensed under a Creative Commons Attribution 4.0 license (http://creativecommons.org/ licenses/by/4.0/). 
Before we proceed, we should make an important, general observation. The familiar tried-and-tested method for analyzing any problem in fluid mechanics is to work, first, from the full set of governing equations. The challenge is then to produce a suitable solution, either by using asymptotic methods [see the recent survey in Johnson (2018)] or, exceptionally, by constructing an exact solution [see Constantin $(2012,2014)$ and Henry (2016) for exact solutions in the Lagrangian framework and Constantin and Johnson (2016) for exact solutions in the Eulerian setting]. In any event, the result is certainly robust, coherent and reliable, but idealized. When applied to a very specific flow configuration, it might fail to describe the details of what is observed and measured. In the context of oceanic flows, this could be due to the action of turbulent mixing, interaction with additional currents, bottom topography, and much more. Nevertheless, we contend that the synoptic structure of the flow will be accurately represented by a suitable solution of the fluid system. Only when this has been found, and analyzed, can we begin the process, often based on modeling ideas, of adding the ingredients which will produce a description that gets closer to the flows that we observe and measure in nature. The purpose of our presentation here is to provide a starting point for a careful treatment of EUC-type flows, by providing a firm theoretical basis. One important bonus in our formulation is that there is much freedom in the choices that can be made, allowing the possibility of further investigation, hopefully leading to more accurate and realistic representations of the flow field; in addition, it provides the opportunity to apply the same principles to other flows.

\section{Governing equations}

We introduce a set of (right-handed) spherical coordinates $\left(\varphi, \theta, r^{\prime}\right): r^{\prime}$ is the distance (radius) from the center of the spherical Earth, $\theta \in[-\pi / 2, \pi / 2]$ is the angle of latitude, and $\varphi \in[0,2 \pi)$ is the azimuthal angle, that is, the angle of longitude. (We use primes throughout the formulation of the problem to denote physical (dimensional) variables; these will be removed when we nondimensionalize.) The unit vectors in this $\left(\varphi, \theta, r^{\prime}\right)$ system, for all points but the two poles, are $\left(\mathbf{e}_{\varphi}, \mathbf{e}_{\theta}, \mathbf{e}_{r}\right)$, respectively, and the corresponding velocity components are $\left(u^{\prime}, v^{\prime}, w^{\prime}\right) ; \mathbf{e}_{\varphi}$ points from west to east, $\mathbf{e}_{\theta}$ from south to north, and $\mathbf{e}_{r}$ upward (see Fig. 1). The $\left(\varphi, \theta, r^{\prime}\right)$ system is associated with a point fixed on the sphere (other than the two poles, where this system is not adequate since $\mathbf{e}_{\varphi}$ and $\mathbf{e}_{\theta}$ are not well defined there), which is rotating about its polar axis with (constant) angular speed $\Omega^{\prime} \approx 7.29 \times 10^{-5} \mathrm{rad} \mathrm{s}^{-1}$.

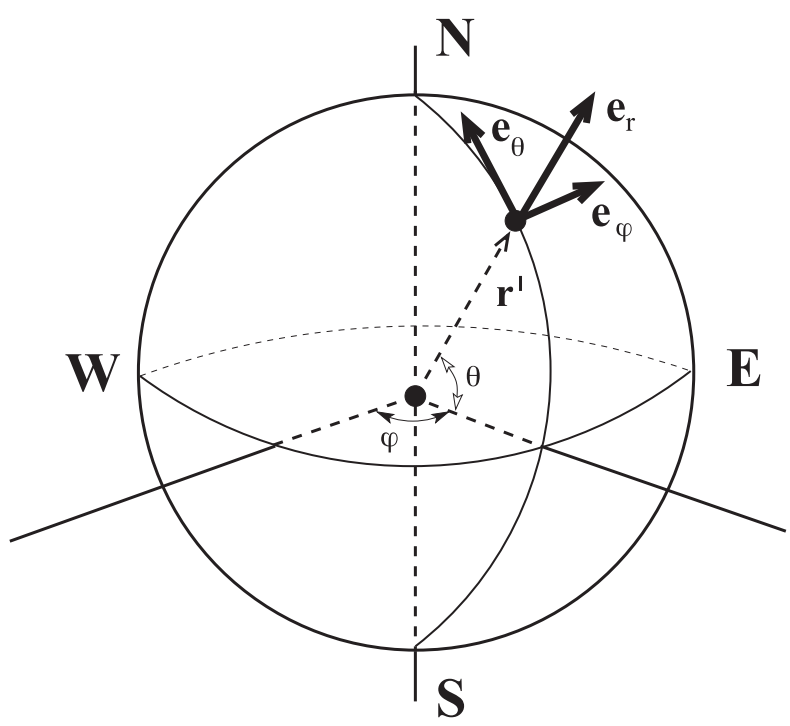

FIG. 1 . The spherical coordinate system, with $\theta \in[-\pi / 2, \pi / 2]$ the angle of latitude, $\varphi \in[0,2 \pi)$ the azimuthal angle (the angle of longitude), and $\mathbf{r}^{\prime}$ the position vector relative to the origin.

To proceed, we first redefine the pressure $p^{\prime}$ so that it is measured relative to the gravitational and centripetal contributions:

$$
p^{\prime}=\rho^{\prime}\left(-g^{\prime} r^{\prime}+\frac{1}{2} r^{\prime 2} \Omega^{\prime 2} \cos ^{2} \theta\right)+P^{\prime}\left(\varphi, \theta, r^{\prime}, t^{\prime}\right),
$$

where $\rho^{\prime}$ is the constant density and $g^{\prime}=$ constant $\approx$ $9.81 \mathrm{~m} \mathrm{~s}^{-2}$. Assuming constant $g^{\prime}$ is reasonable for the depths of the oceans on Earth. We write

$$
r^{\prime}=R^{\prime}+z^{\prime}
$$

where $R^{\prime} \approx 6378 \mathrm{~km}$ is the (mean) radius of Earth and then nondimensionalize according to

$$
z^{\prime}=D^{\prime} z, \quad\left(u^{\prime}, v^{\prime}, w^{\prime}\right)=U^{\prime}(u, v, k w), \quad P^{\prime}=\rho^{\prime} U^{\prime 2} P,
$$

where $D^{\prime}$ is an appropriate depth scale, $U^{\prime}$ a suitable speed scale, and $k$ a scaling factor (which can be used to characterize different classes of flows). The depth scale, in this context, is most appropriately chosen to be a measure of the depth to the bottom of the EUC; we introduce the parameter

$$
\varepsilon=D^{\prime} / R^{\prime}
$$

Because we assume that the velocity profile associated with the EUC is given and so maintained [being, basically, wind-induced and balanced by the Coriolis force, featuring strongly nonlinear effects; see the discussions in Boyd (2018) and Constantin and Johnson (2019)], we may proceed by assuming a steady inviscid fluid flow, and so we use 
the Euler equation for steady flow. Further, the extra detail afforded by the inclusion of any density stratification, or the presence of a thermocline, is irrelevant to the overall structure of the underlying currents; the thermocline can be added to the model, once the velocity field is established. Thus, we take the governing equations for steady flow to be

$$
\begin{aligned}
& {\left[\frac{u}{(1+\varepsilon z) \cos \theta} \frac{\partial}{\partial \varphi}+\frac{v}{1+\varepsilon z} \frac{\partial}{\partial \theta}+\frac{k}{\varepsilon} w \frac{\partial}{\partial z}\right](u, v, k w)} \\
& \quad+\frac{1}{1+\varepsilon z}\left(-u v \tan \theta+k u w, u^{2} \tan \theta+k v w,-u^{2}-v^{2}\right) \\
& \quad+2 \omega(-v \sin \theta+k w \cos \theta, u \sin \theta,-u \cos \theta) \\
& \quad=-\left[\frac{1}{(1+\varepsilon z) \cos \theta} \frac{\partial P}{\partial \varphi}, \frac{1}{1+\varepsilon z} \frac{\partial P}{\partial \theta}, \frac{1}{\varepsilon} \frac{\partial P}{\partial z}\right]
\end{aligned}
$$

and

$$
(1+\varepsilon z)\left[\frac{\partial u}{\partial \varphi}+\frac{\partial}{\partial \theta}(v \cos \theta)\right]+\frac{k}{\varepsilon} \cos \theta \frac{\partial}{\partial z}\left[(1+\varepsilon z)^{2} w\right]=0,
$$

where we have introduced the additional parameter

$$
\omega=\Omega^{\prime} R^{\prime} / U^{\prime},
$$

the inverse Rossby number based on the chosen scales.

The boundary conditions, expressed in the appropriate nondimensional form, become, at the free surface,

$$
\begin{aligned}
P & =P_{s}(\varphi, \theta) \quad \text { and } \\
\frac{k w}{\varepsilon} & =\frac{u}{(1+\varepsilon h) \cos \theta} \frac{\partial h}{\partial \varphi}+\frac{v}{1+\varepsilon h} \frac{\partial h}{\partial \theta} \quad \text { on } \\
z & =h(\varphi, \theta),
\end{aligned}
$$

that is, the pressure and kinematic conditions, respectively, and if a stationary, impermeable bottom boundary is prescribed, then

$$
\frac{k w}{\varepsilon}=\frac{u}{(1+\varepsilon b) \cos \theta} \frac{\partial b}{\partial \varphi}+\frac{v}{1+\varepsilon b} \frac{\partial b}{\partial \theta} \quad \text { on } \quad z=b(\varphi, \theta),
$$

although it is sufficient here to invoke conditions of either an abyssal, stagnant ocean below the surface layer or a lower region where the horizontal flow decays (allowing, therefore, any suitable upwelling or downwelling).

\section{A general observation about equatorial three-dimensional flows}

We now show that excluding any variation in $\varphi$ produces a solution that is not consistent with the flow structure that is observed in the neighborhood of the equator. For ease of presentation (and to be consistent with what follows), we choose $k=\varepsilon$; this is the natural choice within the shallow-water approximation (although the precise choice of $k$ is irrelevant to the development that we present in this section). The issue that we address here is whether there exist solutions without variations in the azimuthal direction-so depending only on $(\theta, z)$-which capture the salient features of flows along the equator, namely,

(i) the meridional surface flow is divergent (poleward) from the line of the equator [i.e., $\operatorname{sgn}(v)=\operatorname{sgn}(\theta)$ near $\theta=0$ on the spherical surface $z=0$ ],

(ii) near-surface upwelling occurs all along the equator (i.e., in the plane $\theta=0$, we have $w>0$ for $z_{0}<z<0$, with $z_{0}$ corresponding to about 300400-m depth), and

(iii) zonal flow reversal occurs in the near-surface all along the equator, with an eastward jet beneath a westward surface flow and above a practically still abyssal layer [i.e., in the vertical plane $\theta=0, u$ increases from a minimal value $u<0$ at $z=0$ to a maximal value $u>0$ at the depth $z_{1} \in\left(z_{0}, 0\right)$ of the core of the EUC, with $u$ decaying rapidly at greater depth],

as highlighted by field data gathered in the Pacific (see Figs. 2-4). The equations of mass conservation and the $\varphi$ component of Euler's equation become

$(1+\varepsilon z) \frac{\partial}{\partial \theta}(v \cos \theta)+\frac{\partial}{\partial z}\left[(1+\varepsilon z)^{2} w \cos \theta\right]=0, \quad$ and

$$
\begin{aligned}
& v\left[\frac{1}{1+\varepsilon z}\left(\frac{\partial u}{\partial \theta}-u \tan \theta\right)-2 \omega \sin \theta\right] \\
& +w\left(\frac{\partial u}{\partial z}+\frac{\varepsilon u}{1+\varepsilon z}+2 \varepsilon \omega \cos \theta\right)=0,
\end{aligned}
$$

respectively.

First, if we invoke the oft-used assumption that $w=0$ for these flows [based on the observation that the ratio of vertical speed to horizontal speed is typically less than $10^{-4}$; see the field data for the Pacific provided in Johnson et al. (2001)], then Eq. (8) shows that $v(\theta, z)=F(z) / \cos \theta$, where $F(z)$ is an arbitrary function. This prevents $v \rightarrow 0$ as $\theta \rightarrow 0$ unless $v \equiv 0$ throughout the equatorial flow region. We deduce, therefore, that for the constraint $v=0$ on $\theta=0$ in point (i) above to hold, we must retain $w$.

We now, second, consider the form taken by Eqs. (8) and (9) as $\theta \rightarrow 0$. The flow in the azimuthal direction on $\theta=0$ is written as $u=u_{0}(z)$. Then Eq. (8) becomes

$$
(1+\varepsilon z) \frac{\partial v}{\partial \theta}+\frac{\partial}{\partial z}\left[(1+\varepsilon z)^{2} w\right]=0 \quad \text { on } \quad \theta=0,
$$

and from Eq. (9), we observe that 


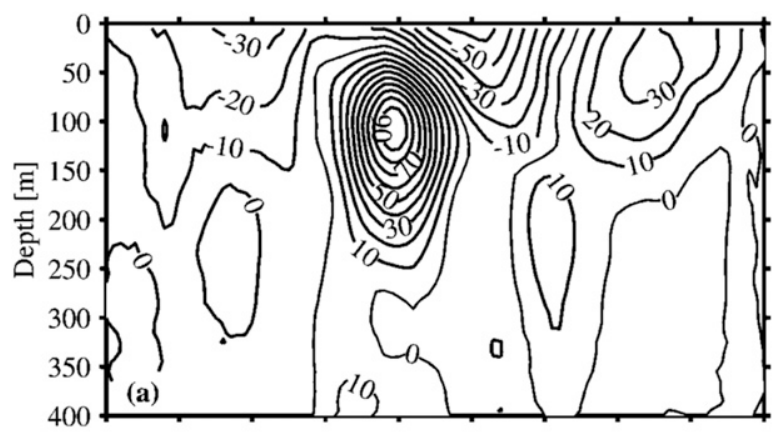

$$
\begin{array}{lllllllll}
8^{\circ} \mathrm{S} & 6^{\circ} \mathrm{S} & 4^{\circ} \mathrm{S} & 2^{\circ} \mathrm{S} & \begin{array}{l}
0^{\circ} \\
\text { Latitude }
\end{array} 2^{\circ} \mathrm{N} & 4^{\circ} \mathrm{N} & 6^{\circ} \mathrm{N} & 8^{\circ} \mathrm{N} & 10^{\circ} \mathrm{N}
\end{array}
$$

FIG. 2. Typical level sets of the zonal velocity $u\left(10^{-2} \mathrm{~m} \mathrm{~s}^{-1}\right)$ at a fixed longitude in the equatorial Pacific, based on shipboard observations. Reproduced from Johnson et al. (2001).

$$
\frac{\partial u}{\partial z}+\frac{\varepsilon u}{1+\varepsilon z}+2 \varepsilon \omega \cos \theta \rightarrow \frac{d u_{0}}{d z}+\frac{\varepsilon u_{0}}{1+\varepsilon z}+2 \varepsilon \omega
$$

$$
\text { as } \theta \rightarrow 0
$$

and

$$
\begin{aligned}
& \frac{1}{1+\varepsilon z}\left(\frac{\partial u}{\partial \theta}-u \tan \theta\right)-2 \omega \sin \theta \rightarrow \frac{1}{1+\varepsilon z} u_{d}(z) \quad \text { as } \\
& \theta \rightarrow 0
\end{aligned}
$$

where $\partial u / \partial \theta \rightarrow u_{d}(z)$ as $\theta \rightarrow 0$ [and $u_{d}(z)$ might be zero- this certainly being the case if $u$ presents meridional symmetry about the equator]. Using these two results, together with $v=0$ on $\theta=0$, Eq. (9) gives

$$
w\left[\frac{d u_{0}}{d z}+\frac{\varepsilon u_{0}(z)}{1+\varepsilon z}+2 \varepsilon \omega\right]=0 \quad \text { on } \quad \theta=0 .
$$

Since $w(0, z)>0$ for $z>z_{0}$, by virtue of point (ii), Eq. (11) yields

$$
\frac{d u_{0}}{d z}+\frac{\varepsilon u_{0}(z)}{1+\varepsilon z}+2 \varepsilon \omega=0, \quad z_{0}<z<0,
$$

and so

$$
u_{0}(z)=\frac{c}{1+\varepsilon z}-\omega(1+\varepsilon z), \quad z_{0}<z<0,
$$

which, with $u_{0}(0)<0$ given (and hence fixing the arbitrary constant $c$ ), therefore prescribes the zonal velocity profile. However, this profile does not describe a profile which has a positive maximum below the surface, at $z=z_{1}<0$, and which thereafter decays with increasing depth, as required to recover the observational data for the EUC-see item (iii) above. Indeed, $d u_{0} / d z=0$ at $z=z_{1}$ yields $c=-\omega\left(1+\varepsilon z_{1}\right)^{2}$, and then $u_{0}\left(z_{1}\right)=-2 \omega\left(1+\varepsilon z_{1}\right)<0$.

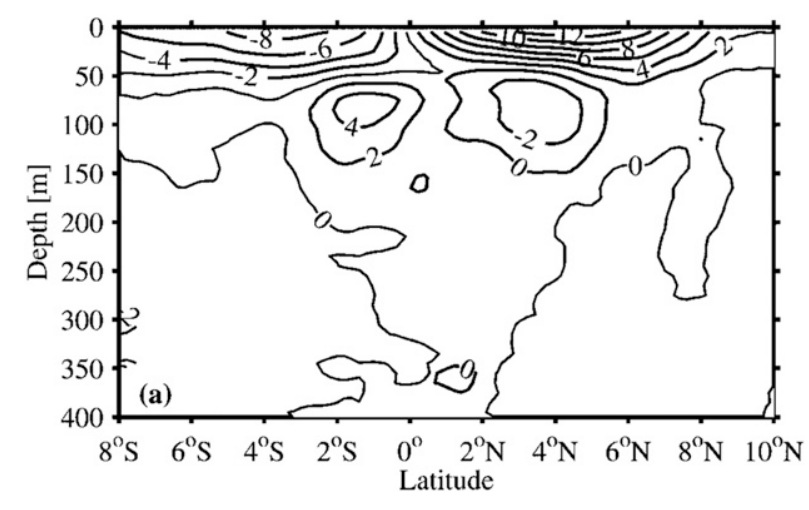

FIG. 3. Typical level sets of the meridional velocity $v\left(10^{-2} \mathrm{~m} \mathrm{~s}^{-1}\right)$ at a fixed longitude in the equatorial Pacific, based on shipboard observations. Reproduced from Johnson et al. (2001).

On the other hand, let us allow a variation in the azimuthal direction, but set $w=0$; then Eq. (4) becomes

$$
\frac{\partial u}{\partial \varphi}+\frac{\partial}{\partial \theta}(v \cos \theta)=0
$$

which implies a streamfunction representation:

$$
u=\frac{\partial \psi}{\partial \theta}, \quad v=-\frac{1}{\cos \theta} \frac{\partial \psi}{\partial \varphi} .
$$

Then the kinematic condition at the surface in Eq. (6) shows that the free surface can be expressed as $h=h(\psi)$, that is, it is constant on the streamlines associated with the flow below the surface. Although this is certainly a possible solution, it cannot admit more general choices of the shape of the free surface controlled, for example, by a suitable pressure distribution over the surface.

In summary, we must conclude that, for flows which correspond to what is observed in the neighborhood of the equator in the Pacific, we necessarily require both $w \neq 0$ and evolution in the azimuthal direction. We also anticipate that nonlinearity will play an essential role, since, as shown in Henry (2018), linearization fails to capture the large coherent structures that are representative of the upwelling and poleward near-surface meridional flow processes observed in equatorial regions (as illustrated in Figs. 3 and 4). Direct measurements also confirm the nonlinear dynamics of the Pacific equatorial flow (see Kessler et al. 2003).

\section{Asymptotic structure}

The overarching principle that drives our analysis is the shallow-water (thin shell) approximation. Thus with the choice $k=\varepsilon$, we invoke $\varepsilon \rightarrow 0$ (and for guidance, a typical value of $\varepsilon$ is about $10^{-5}$ ). However, 


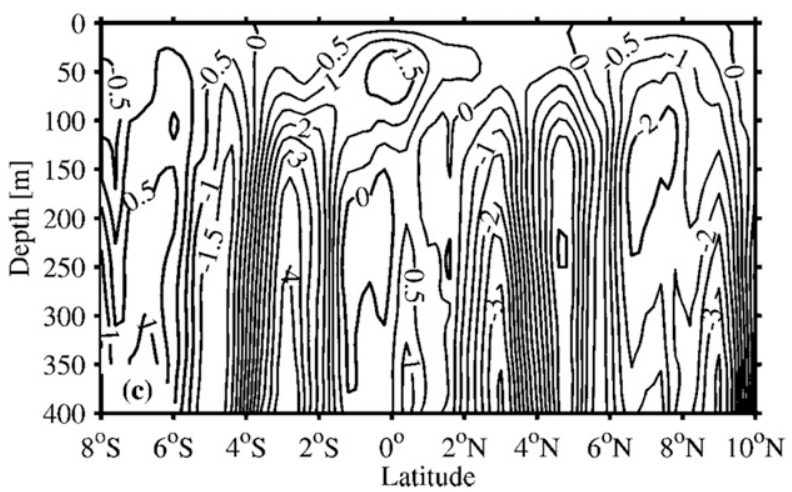

FIG. 4. Typical level sets of the vertical velocity $w\left(10^{-5} \mathrm{~m} \mathrm{~s}^{-1}\right)$ at a fixed longitude in the equatorial Pacific, based on shipboard observations. Reproduced from Johnson et al. (2001).

we also note that $\omega$ is fairly large (about 400, based on values appropriate for the Pacific EUC) and so we introduce a second limit process: $\omega \rightarrow \infty$, but such that $\varepsilon \omega \rightarrow 0$. [This choice of limiting process implies that a coherent mathematical description requires $\omega=\omega(\varepsilon)$, even though these are two independent parameters in the underlying physical problem.] With this choice of the limit processes, we see that Eqs. (3), (4), and (6) give, at leading order in $\varepsilon$,

$$
\begin{aligned}
\frac{u}{\cos \theta} \frac{\partial u}{\partial \varphi}+v \frac{\partial u}{\partial \theta}+w \frac{\partial u}{\partial z}-u v \tan \theta-2 \omega v \sin \theta & =-\frac{\partial P}{\partial \varphi}, \\
\frac{u}{\cos \theta} \frac{\partial v}{\partial \varphi}+v \frac{\partial v}{\partial \theta}+w \frac{\partial v}{\partial z}+u^{2} \tan \theta+2 \omega u \sin \theta & =-\frac{\partial P}{\partial \theta}, \\
\frac{\partial P}{\partial z} & =0,
\end{aligned}
$$

and

$$
\frac{\partial u}{\partial \varphi}+\frac{\partial}{\partial \theta}(v \cos \theta)+\cos \theta \frac{\partial w}{\partial z}=0,
$$

with

$$
\begin{aligned}
P & =P_{s}(\varphi, \theta) \quad \text { and } \quad w=\frac{u}{\cos \theta} \frac{\partial h}{\partial \varphi}+v \frac{\partial h}{\partial \theta} \quad \text { on } \\
z & =h(\varphi, \theta) .
\end{aligned}
$$

(We will include the appropriate conditions below the surface layer later.)

This set of equations is valid for any meridional angle $\theta$; this is therefore the relevant system for describing the flows for $\theta=O(1)$, that is, well away from the equator. Then, with the condition that $\omega$ is large, we see that the leading order consistent with Eqs. (12) and (13) implies that both $u$ and $v$ must be small [i.e., $O(1 / \omega)$ ] although $w$ need not be. However, we are specifically concerned with the solution of Eqs. (12)-(16) in the neighborhood of the equator $(\theta=0)$, and so we examine these equations for small $\theta$. Our primary interest here is with the solution of Eqs. (12)-(16) in the neighborhood of the equator (which is on $\theta=0$ ), and so we examine these equations for small $\theta$, coupled with large $\omega$. In particular, we are interested in the region where the EUC and the westward near-surface flow exist, and so we introduce $\theta=\delta \Theta$, and examine the problem defined by $\delta \rightarrow 0$; this leads to the identification of two regions. The outermost region, which essentially defines the maximum extent of the EUC structure, is described by the choice $\delta=1 / \sqrt{\omega}$ with

$$
v=\frac{1}{\sqrt{\omega}} V(\varphi, \Theta, z)
$$

(necessary for consistency) and

$$
\begin{array}{ll}
u=U(\varphi, \Theta, z), \quad w=W(\varphi, \Theta, z), \\
P=\Pi(\varphi, \Theta, z), \quad h=H(\varphi, \Theta) .
\end{array}
$$

The leading order, as $\omega \rightarrow \infty$, gives

$$
\begin{aligned}
U \frac{\partial U}{\partial \varphi}+V \frac{\partial U}{\partial \Theta}+W \frac{\partial U}{\partial z}-2 \Theta V & =-\frac{\partial \Pi}{\partial \varphi}, \\
\frac{\partial \Pi}{\partial \Theta} & =-2 \Theta U, \\
\frac{\partial \Pi}{\partial z} & =0,
\end{aligned}
$$

and

$$
\frac{\partial U}{\partial \varphi}+\frac{\partial V}{\partial \Theta}+\frac{\partial W}{\partial z}=0,
$$

with

$$
\begin{aligned}
\Pi & =\hat{\Pi}(\varphi, \Theta) \quad \text { and } \quad W=U \frac{\partial H}{\partial \varphi}+V \frac{\partial H}{\partial \Theta} \quad \text { on } \\
z & =H(\varphi, \Theta) .
\end{aligned}
$$

This system is satisfied only if $\partial U / \partial z=0$; indeed, the available data indicate that we could take $U=0$ in this region. On the other hand, the variation of $U$ (inherent in the description of the EUC structure) occurs within an inner region; this is defined by any choice of $\delta$ which satisfies $\delta \sqrt{\omega} \rightarrow 0$, that is, $\delta=o(1 / \sqrt{\omega})$ as $\omega \rightarrow \infty$. (The natural interpretation here is that the prescribed $U$ profile, at some given $\varphi$, fixes the parameter $\delta$.) Now with

$$
\begin{aligned}
v & =\delta V(\varphi, \Theta, z), \quad u=U(\varphi, \Theta, z), \quad w=W(\varphi, \Theta, z), \\
P & =\Pi(\varphi, \Theta, z), \quad h=H(\varphi, \Theta),
\end{aligned}
$$


and retaining the leading order in this limit, reduces Eqs. (12)-(16) to

$$
\begin{aligned}
U \frac{\partial U}{\partial \varphi}+V \frac{\partial U}{\partial \Theta}+W \frac{\partial U}{\partial z} & =-\frac{\partial \Pi}{\partial \varphi}, \\
\frac{\partial \Pi}{\partial \Theta} & =0 \\
\frac{\partial \Pi}{\partial z} & =0
\end{aligned}
$$

and

$$
\frac{\partial U}{\partial \varphi}+\frac{\partial V}{\partial \Theta}+\frac{\partial W}{\partial z}=0
$$

with

$$
\begin{aligned}
& \Pi=\hat{\Pi}(\varphi), \\
& W=U \frac{\partial H}{\partial \varphi}+V \frac{\partial H}{\partial \Theta} \quad \text { on } \quad z=H(\varphi, \Theta),
\end{aligned}
$$

where we must have $\Pi=\Pi(\varphi)$ only, to satisfy the constraints imposed by Eqs. (18) and (19). It is the solution of this system that is of most interest to us, since it provides a consistent description of the velocity structure in the neighborhood of the equator. Although we could readily reduce the original system to this simplified version of the problem by invoking simple approximation arguments, the approach adopted here enables us to be precise about the approximation and its validity. Further, and of some significance, it opens the way to generating higherorder terms, if these are deemed necessary. This would be a natural development if numerical solutions are being considered, enabling the construction of a more accurate approximation. There is no hint of nonuniformities in the double asymptotic expansions, based on $\varepsilon$ and $\delta$, needed in this problem, provided that we restrict the interpretation to $\Theta=O(1)$ in the outer $[\theta=O(1 / \sqrt{\omega})]$ region around the line of the equator. Indeed, with $\theta=\delta \Theta$ (for $\delta \sqrt{\omega} \rightarrow 0$ ), we could limit the EUC solution to the region described by $|\Theta| \leq 1$, with the zero solution in $|\Theta|>1$. If we do allow $\theta$ to increase beyond $O(1 / \sqrt{\omega})$, then we must match to a suitable solution of Eqs. (12)-(16), taking into account, for example, the remarkably steady subsurface countercurrents (the Tsuchiya jets) which bracket the EUC, from about $5^{\circ}$ either side of the equator (see Rowe et al. 2000). However, our main concern in this analysis is with the region close to the equator, that is, for $\theta=\delta \Theta,|\Theta| \leq 1$, with $\delta \sqrt{\omega} \rightarrow 0$.

\section{Method of solution for the inner region}

The development that we present follows Constantin and Johnson (2017), but the underlying flow is more completely and accurately described here. First, from Eqs. (18), (19), and (21a), we see that

$$
\Pi=\hat{\Pi}(\varphi),
$$

and then the elimination of $\partial U / \partial \varphi$ between Eqs. (17) and (20) gives

$$
-U\left(\frac{\partial V}{\partial \Theta}+\frac{\partial W}{\partial z}\right)+V \frac{\partial U}{\partial \Theta}+W \frac{\partial U}{\partial z}=-\hat{\Pi}^{\prime},
$$

where the prime denotes the derivative with respect to $\varphi$. Thus, we have

$$
\frac{\partial}{\partial \Theta}\left(\frac{V}{U}\right)+\frac{\partial}{\partial z}\left(\frac{W}{U}\right)=\frac{\hat{\Pi}^{\prime}}{U^{2}} .
$$

Introducing a pseudostreamfunction $\psi(\varphi, \Theta, z)$ such that

$$
\frac{V}{U}=\frac{\partial \psi}{\partial z}
$$

gives

$$
\frac{W}{U}=-\frac{\partial \psi}{\partial \Theta}-\hat{\Pi}^{\prime}(\varphi) \int_{z}^{H(\varphi, \Theta)} \frac{d z^{\prime}}{U^{2}\left(\varphi, \Theta, z^{\prime}\right)}
$$

where any additive arbitrary function of $(\varphi, \Theta)$ is redundant because it vanishes identically when we introduce the relevant solution for $\psi(\varphi, \Theta, z)$. We now use Eqs. (23) and (24) in Eq. (17) to give

$$
U_{\Theta} \psi_{z}-U_{z} \psi_{\Theta}=\hat{\Pi}^{\prime} \frac{\partial}{\partial z}\left\{U \int_{z}^{H} \frac{d z^{\prime}}{U^{2}}\right\}-U_{\varphi},
$$

where subscripts denote partial derivatives; the solution can be expressed as

$$
\psi=\Psi(\varphi, \Theta, U),
$$

which gives

$$
\Psi_{\Theta}=\frac{1}{U_{z}}\left[U_{\varphi}-\hat{\Pi}^{\prime} \frac{\partial}{\partial z}\left(U \int_{z}^{H} \frac{d z^{\prime}}{U^{2}}\right)\right] .
$$

To proceed, we suppose, in this initial investigation, that we may write

$$
U=\hat{U}(\zeta, \varphi),
$$

where $\zeta=f(\varphi, \Theta, z)$, which will play the role similar to that of a characteristic variable; then we seek a solution of Eq. (25) in the form 


$$
\Psi=\hat{\Psi}(\varphi, \Theta, \zeta)
$$

and so Eq. (25) becomes

$$
f_{z} \hat{\Psi}_{\Theta}=f_{\varphi}+\frac{1}{\hat{U}_{\zeta}}\left[\hat{U}_{\varphi}-\hat{\Pi}^{\prime} \frac{\partial}{\partial z}\left(U \int_{z}^{H} \frac{d z^{\prime}}{U^{2}}\right)\right] .
$$

Finally, we note, from Eq. (17), that if there is a region where $U \equiv 0$ (as it is the case outside the region occupied by the EUC structure, for example, and we will invoke this model here), then we must have $\hat{\Pi}=$ constant; thus, we obtain

$$
\begin{aligned}
\hat{\Psi}_{\Theta} & =\frac{G}{F}+\frac{\hat{U}_{\varphi}}{\hat{U}_{\zeta} F}, \text { where } f_{\varphi}=F(\varphi, \Theta, \zeta), \\
f_{\Theta} & =G(\varphi, \Theta, \zeta),
\end{aligned}
$$

that is,

$$
\hat{\Psi}(\varphi, \Theta, \zeta)=\int_{0}^{\Theta}\left[G\left(\varphi, \Theta^{\prime}, \zeta\right)+\frac{\hat{U}_{\varphi}(\varphi, \zeta)}{\hat{U}_{\zeta}(\varphi, \zeta)}\right] \frac{d \Theta^{\prime}}{F\left(\varphi, \Theta^{\prime}, \zeta\right)} .
$$

This particular version of the solution is chosen so that $V=0$ on $\Theta=0$ and thus, by virtue of Eq. (23), $V=0$ on $\theta=0$. Furthermore, we note that $\hat{\Psi}=0$ if there is no $\varphi$ dependence in the azimuthal direction (consistent with the general argument presented in section 3). While in principle any of the three velocity components $(U, V, W)$ determines the other two, it is more natural to start with $U$, and then find $V$ and $W$, as above. Indeed, there is symmetry in the way that $V$ and $W$ enter the problem, which is not repeated by a pairing that involves $U$. Nevertheless, assuming that $V$ is given, Eq. (20) can be solved by introducing a pseudostreamfunction

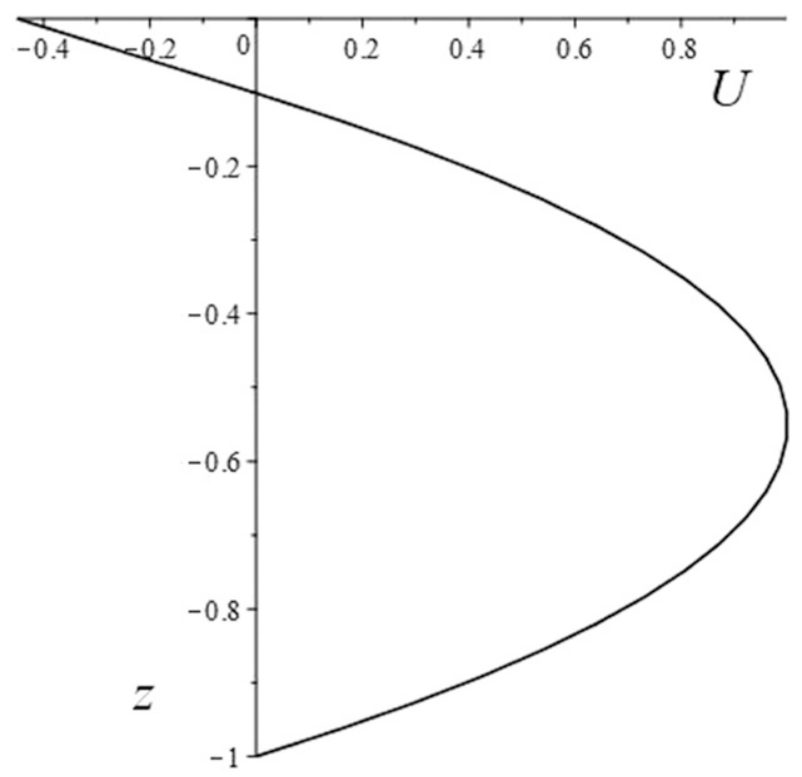

FIG. 5. Plot of a typical velocity profile vertically below the equator.

describing $U$ and $W$, and then Eq. (17) yields a nonlinear equation for this new streamfunction. However, this development is considerably more involved than the approach described above.

\section{A model for the Pacific equatorial flow}

The plan now is to show how the general formulation from section 5 can be used to describe the details of flows that follow the pattern of the Pacific Equatorial Undercurrent. To this end, we first make a suitable (but simple) choice for $U(0,0, z)$ that will provide the basis for the solution that we present here; we write

$$
U(0,0, z)=1-\left\{\frac{\left[1-u_{s}-\left(\frac{z_{0}}{R_{0}}\right)^{4}\right]\left(\frac{z+z_{0}}{R_{0}}\right)^{2}-\left[1-u_{s}-\left(\frac{z_{0}}{R_{0}}\right)^{2}\right]\left(\frac{z+z_{0}}{R_{0}}\right)^{4}}{\left(\frac{z_{0}}{R_{0}}\right)^{2}\left[1-\left(\frac{z_{0}}{R_{0}}\right)^{2}\right]}\right\}
$$

which corresponds to the choice that was made in Constantin and Johnson (2017)—apparently the simplest choice that satisfies all the requirements for these types of flow. This profile (shown in Fig. 5 for the case $z_{0}=0.6$, $\left.R_{0}=0.5, u_{s}=-0.5\right)$ has the properties $U\left(0,0,-z_{0}\right)=1$, $U(0,0,0)=u_{s}$, and $U\left(0,0,-z_{0} \pm R_{0}\right)=0$, implying that the chosen scale speed $U^{\prime}$ is the maximum speed in the undercurrent, and here we have set the surface speed to be half of this (in the opposite direction). Further, the scale length $D^{\prime}$ is the depth to the bottom of the EUC.

This profile must now be embedded in a region in the neighborhood of the equator, allowing for a structure in the vertical, meridional and azimuthal directions. This is accomplished by first introducing suitable choices for the "characteristic" variable $(\zeta=f)$ : 


$$
f(\varphi, \Theta, z)=\left\{\begin{array}{c}
{\left[z+z_{0}(\varphi)\right]^{2}+\lambda^{2} \Theta^{2},} \\
{\left[z+z_{0}(\varphi)\right]^{2}+\lambda^{2} \Theta^{2}-\mu \lambda^{2}\left(\Theta \mp \Theta_{0}\right)^{2},}
\end{array}\right.
$$

in the EUC core and in the surface layer for $|\Theta| \leq \Theta_{0}$, in the surface layer for $|\Theta|>\Theta_{0}$, where $\lambda, \mu$, and $\Theta_{0}$ are positive constants (and signs ordered according to $\Theta>\Theta_{0}, \Theta<-\Theta_{0}$ ), and then writing

$$
\hat{U}(\varphi, \zeta)=\alpha(\varphi)-\beta(\varphi) \zeta+\gamma(\varphi) \zeta^{2},
$$

with $\zeta=\sqrt{R(\varphi)}$ on the zero-boundary of the EUC-core, that is, $\alpha-\beta R^{2}+\gamma R^{4}=0$. Using these choices we have a typical profile [based on Eq. (28), with $\mu=2.5$ and $\left.\lambda \Theta_{0}=0.35\right]$ represented in Fig. 6 as a contour plot in the $(X, z)$ plane, where $X=\lambda \Theta$. Further, we assume that the component of the velocity in the azimuthal direction is zero outside the region portrayed in Fig. 6; this general structure corresponds to the available data for flow in the EUC [see, e.g., Johnson et al. (2001), as shown in Fig. 3]. The consequences of using this basic structure for the Pacific EUC are what we now examine. However, in this initial discussion, we will not introduce any specific evolution in the azimuthal direction - this is planned as a future investigation — but, as we have already seen, there must be some evolution for these solutions to exist, although the details are irrelevant at this stage. Thus we will limit our presentation to the form taken by the three-dimensional flow field at some station in the azimuthal direction.

The next stage involves the construction of $\Psi(\varphi, \Theta, z)$, via Eq. (26) with Eqs. (28) and (29); this is a straightforward calculation, producing

$\Psi(\varphi, \Theta, z)=\Theta z_{0}^{\prime}+\frac{1}{2 \lambda}\left(\frac{\alpha-\beta^{\prime} \zeta+\gamma^{\prime} \zeta^{2}}{-\beta+2 \gamma \zeta}\right) \arctan \left(\frac{\lambda \Theta}{z+z_{0}}\right)$,

where $\zeta=\left[z+z_{0}(\varphi)\right]^{2}+\lambda^{2} \Theta^{2}$ and the prime denotes the derivative with respect to $\varphi$; this expression is valid within the core of the EUC and in the surface layer for $-\Theta_{0} \leq \Theta \leq \Theta_{0}$. The existence of a solution requires that $-\beta+2 \gamma \zeta<0$ (because $\beta>0$ and $\zeta=0$ at the center of the EUC core); this term appears in the denominator in Eq. (30) and arises from $\partial \hat{U} / \partial \zeta$ [which is associated with $\left.\partial U / \partial z=(\partial \hat{U} / \partial \zeta) f_{z}\right]$. The velocity components, based on $\Psi_{z}$ and $\Psi_{\Theta}$ [see Eqs. (23) and (24) with $\hat{\Pi}=$ constant], follow directly, although they take a quite complicated form; the relevant information is reproduced in the appendix. One immediate consequence of these formulae is that, in order to avoid the singularity in $\Psi_{z}$ on $z=-z_{0}$ as $\Theta \rightarrow 0$, we must set $\alpha(\varphi)=$ constant; this condition shows that the maximum speed in the EUC core remains constant as the flow evolves in $\varphi$, a condition also found in Constantin and Johnson (2017). A corresponding set of results is obtained from the prescription of the velocity profile in the surface layer where $|\Theta|>\Theta_{0}$; see the appendix.

Finally, these expressions are used in the surface kinematic boundary condition [Eq. (21b)], which (because $U \neq 0$ at the surface) can be written as

$$
\frac{\partial}{\partial \Theta} \Psi[\varphi, \Theta, H(\varphi, \Theta)]+\frac{\partial H}{\partial \varphi}=0 .
$$

If we assume that $H(\varphi, \Theta) \rightarrow H_{0}(\varphi)$ as $\Theta \rightarrow 0$, then Eq. (31) implies that

$$
\beta\left(H_{0}+z_{0}\right)^{2}-\gamma\left(H_{0}+z_{0}\right)^{4}=\text { constant },
$$

which shows that the speed at the surface, along the equator, is constant as the flow evolves in $\varphi$; for a physically realistic solution to exist we must also have $R<H_{0}+z_{0}$. Further, for a flow which moves away from the line of the equator, at least in the surface layer, and for which there is an associated upwelling in the neighborhood of the equator (decreasing in magnitude to zero as the free surface is approached from below), we require

$$
\begin{aligned}
& {\left[\beta-2 \gamma\left(H_{0}+z_{0}\right)^{2}\right] z_{0}^{\prime}} \\
& \quad+\frac{1}{2}\left[\beta^{\prime}-\gamma^{\prime}\left(H_{0}+z_{0}\right)^{2}\right]\left(H_{0}+z_{0}\right)=0
\end{aligned}
$$

and

$$
\begin{aligned}
& \beta \beta^{\prime}-3 \beta \gamma^{\prime}\left(H_{0}+z_{0}\right)^{2}+2 \beta^{\prime} \gamma\left(H_{0}+z_{0}\right)^{2} \\
& \quad+2 \gamma \gamma^{\prime}\left(H_{0}+z_{0}\right)^{4}<0 .
\end{aligned}
$$

Combining Eqs. (32) and (34) shows that there is a solution with $H_{0}(\varphi)=$ constant, which we adopt here, and then choose $H_{0}(\varphi) \equiv 0$. In passing, we observe that the inequality (34) cannot be satisfied if we have a profile which is purely quadratic (i.e., $\gamma \equiv 0$ ) and for which we impose the observed property that the line of maximum speed in the EUC rises to the East (i.e., $z_{0}^{\prime}<0$ ); a corresponding restriction was encountered in Constantin and Johnson (2017). We find that Eq. (34) is satisfied throughout the flow if 


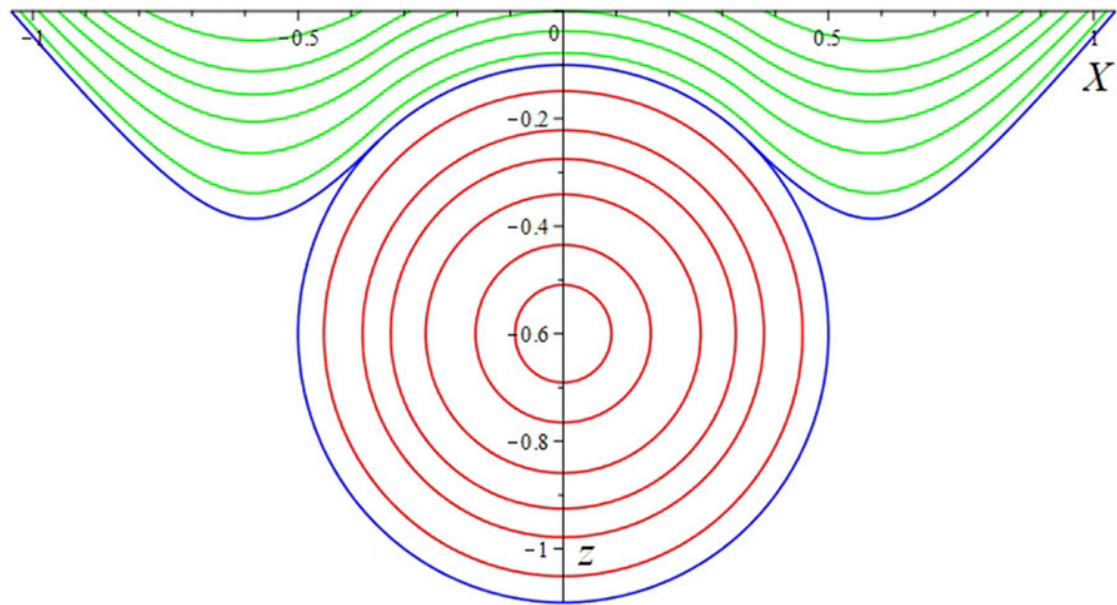

FIG. 6. Contour plot of the azimuthal (nondimensional) velocity component. The blue lines are lines of zero speed; the red contours show the flow toward the east, with a maximum speed of 1 at the center of the circle; the green contours show the flow toward the west, the speed increasing upward, with a speed of 0.5 at the surface on the equator.

$n>3-2 \frac{\gamma z_{0}^{2}}{\beta}, \quad$ where $\quad \frac{\beta^{\prime}}{\beta}=-n(\varphi) \frac{z_{0}^{\prime}}{z_{0}}$.

\section{Some results}

Even a cursory examination of the velocity field described above shows that there are many different types of solutions available, all of which are dynamically possible, but not necessarily observed. Our sole aim in this first analysis of the problem is to ascertain whether solutions of the type found in the neighborhood of the Pacific equator are possible. We present the results as contour plots of speed in the meridional and vertical directions, at fixed $\varphi$, given the profile, $U(\varphi, \Theta, z)$ [obtained from Eqs. (27)-(29) and shown in Fig. 6]. However, in order to minimize the choices that we must make, we elect to plot

$$
\frac{V}{\left(-z_{0}^{\prime} / z_{0}\right)} \text { and } \frac{W}{\left(-z_{0}^{\prime} / z_{0}\right)}
$$

where $z_{0}$ and $-z_{0}^{\prime}$ are both positive (for the EUC); the magnitudes of these (nondimensional) velocity components are therefore recovered only when $z_{0}^{\prime} / z_{0}$ (and $\lambda)$ are specified. (This former observation is yet another indication of the essential role played by the evolution in the azimuthal direction.) The plots are presented with the choice $n=6$; the speed contours are plotted with respect to our coordinates $z$ and $X=\lambda \Theta$.

The velocity component in the meridional direction, across the region where $U \neq 0$, is shown in Fig. 7. This depicts a flow which is away from the equator near the surface, together with two closed regions of opposite polarity a little below the surface; this accords precisely with the data reproduced in Johnson et al. (2001). In addition, in our solution, there are four further regions around the core of the EUC, with suitable reversals of polarity. The corresponding plot for the vertical component of the velocity is shown in Fig. 8. This shows a region of upwelling in the surface layer near the equator and regions of downwelling either side of this; this is followed by a region of upwelling even further away from the equator. In the region occupied by the core of the EUC, we have an area of upwelling surrounded by a region of downwelling. Of course, these results provide no more than a snapshot of what is possible; there are many variants, both in terms of the distribution of regions of positive/negative flows and of upwelling/downwelling. The controlling factor is, predominantly, the choice of the parameter $n$ : as this changes, so the details of the flow field are significantly affected. This is clearly an area for further-and extensive-investigation, particularly with a view to constructing solutions that might relate to other similar flows in the oceans, for example, in the Indian Ocean. The number of regions will depend on the choices of $U$ and the parameter $n$. While the number of regions may vary, the symmetry of $U$ forces a corresponding symmetry (about the vertical through the equator) of the resulting velocity field. In addition, in all the investigations that we undertook, there was always a reversal of polarity across a zero-speed line (which appears to be an intrinsic property of these flows). 


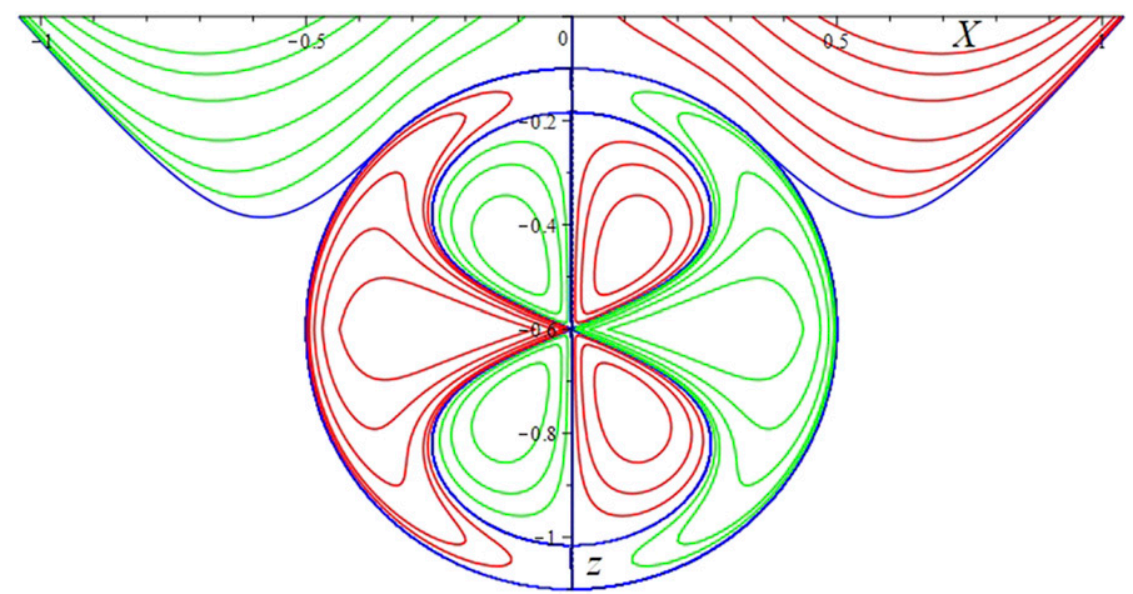

FIG. 7. Contour plot of the meridional (nondimensional) velocity component. The blue lines are lines of zero speed; on the red contours the flow speed is positive and is negative on the green contours. The highest-speed contour shown here in the region near the surface corresponds to 2; within the EUC, the maximum shown in the outer regions is 0.2 and 0.1 in the four inner regions.

We should emphasize that it is quite easy to change the magnitudes of the relative speeds in each plot-we have presented just one (reasonable) possibility that corresponds, more or less, with what is observed in the Pacific EUC. In addition, although the physical speeds for the azimuthal flow (see Fig. 6) can be obtained directly by multiplying the expressions derived from Eqs. (27)-(30) by the speed scale $U^{\prime}$, this principle cannot be applied to the other two velocity components. As we have described, these involve factors that depend on the evolution (in particular, via a first derivative) in the azimuthal direction and, in the case of $V$, also by the scaling factor $\delta$; correspondingly for $W$ we must include the scaling factor $\varepsilon$.
Also, as a contribution to the flow field, we should briefly mention the nature of the flow in the regions where $U=0$, that is, outside the region bounded by the blue lines in Fig. 6. From Eqs. (18)-(20), with $U=0$ and $\Pi_{s}=$ constant, we obtain

$$
\frac{\partial V}{\partial \Theta}+\frac{\partial W}{\partial z}=0
$$

so that in this region there exists a conventional streamfunction, $\tilde{\psi}(\Theta, z)$, where

$$
V=\frac{\partial \tilde{\psi}}{\partial z} \quad \text { and } \quad W=-\frac{\partial \tilde{\psi}}{\partial \Theta} .
$$

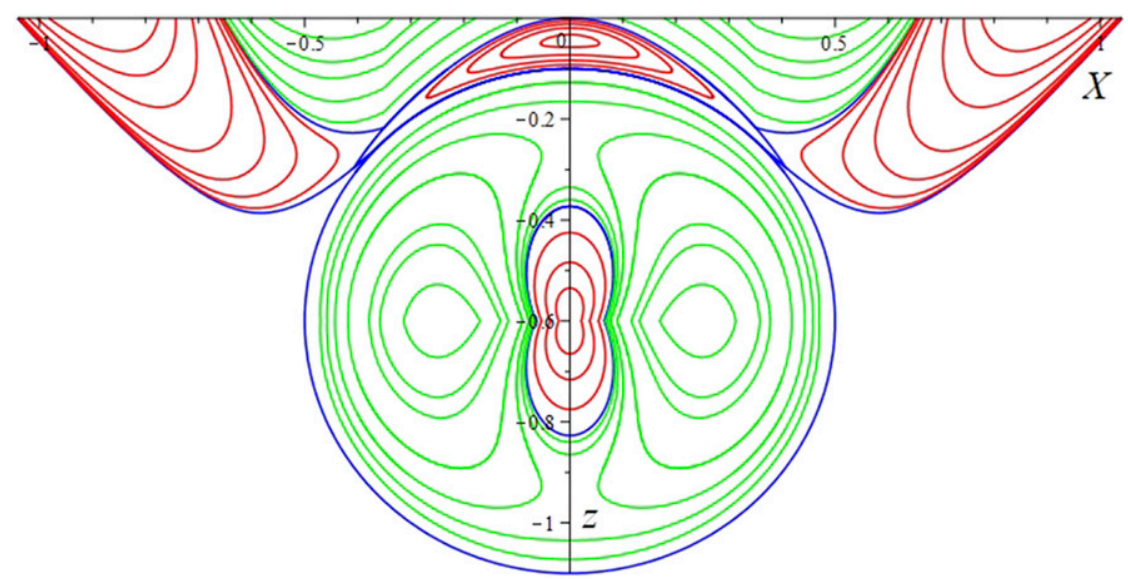

FIG. 8. Contour plot of the vertical (nondimensional) velocity component. The blue lines are lines of zero speed; on the red contours the flow speed is positive (upwelling) and is negative (downwelling) on the green contours. The highest-speed contours shown are, from the center outwards near the surface, 0.07 (upwelling), 1.4 (downwelling), and 2.5 (upwelling); in the core below this, they are 0.7 (downwelling) and 0.05 (upwelling). 


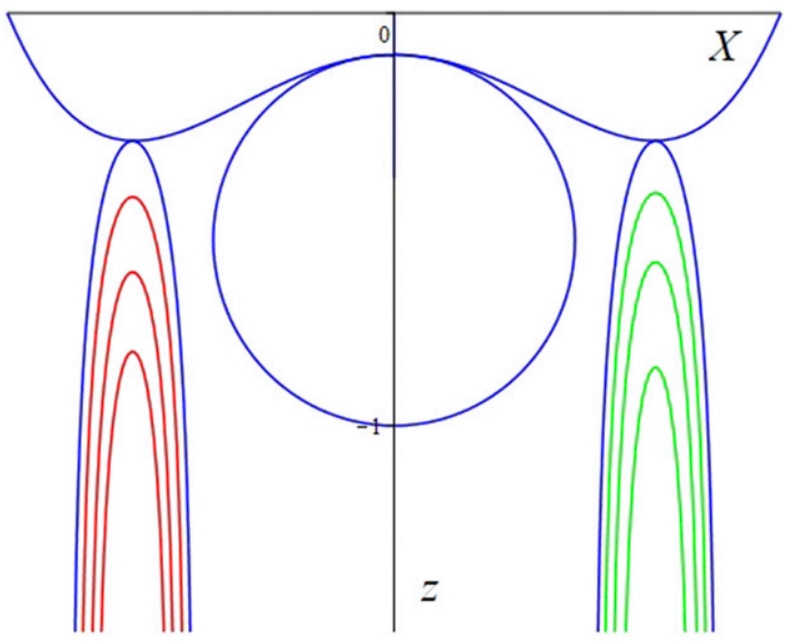

FIG. 9. Plots of two examples of upwelling (red) and downwelling (green), with $\lambda \Theta_{0}= \pm 0.65, z_{1}=0.31$, and $c=0.15$. The blue lines are lines of zero speed; the (nondimensional) speeds on the contours shown here are $0.005,0.01$, and 0.015 (downwelling) and $0.007,0.02$, and 0.04 (upwelling).

These flows can arise independently of any evolution in the azimuthal direction, and so may be superimposed on any solution of the type that we have described above. Guided by the data presented, for example in Johnson et al. (2001) and reproduced in Fig. 4, we assume (for our

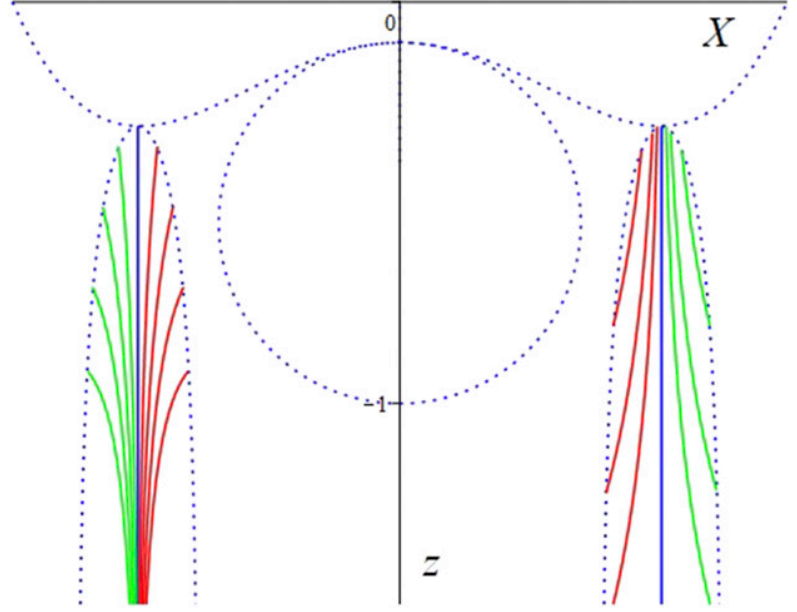

FIG. 10. The meridional (nondimensional) velocity component (red positive, green negative) within the region of (left) upwelling and (right) downwelling. The blue dotted lines are included for guidance, and the heavy blue line is a line of zero speed. The speeds on the contours shown here are $0.0005,0.001$, and 0.002 (downwelling region, right) and 0.1, 0.2, 0.3, and 0.4 (upwelling region, left).

purposes here) that there is some driving mechanism which supports upwelling and downwelling in the region that we have identified as where $U=0$. These types of flows can be represented, for example, by the streamfunction

$$
\tilde{\psi}(\Theta, z)=\left\{\begin{array}{l}
-\frac{\lambda^{3}}{3}\left(\Theta+\Theta_{1}\right)^{3}+c \lambda^{2}\left[1-e^{2\left(z+z_{1}\right)}\right]\left(\Theta+\Theta_{1}\right), \text { (downwelling) } \\
c^{2} \lambda\left(\Theta+\Theta_{1}\right)-c e^{-\left(z+z_{1}\right)} \arctan \left[\frac{\lambda\left(\Theta+\Theta_{1}\right)}{c e^{z+z_{1}}}\right], \text { (upwelling) }
\end{array}\right.
$$

where $c$ is a positive constant which measures the width of the area of upwelling/downwelling, and $\Theta=-\Theta_{1}$ fixes the position of this region. The nondimensional velocity components can be recovered by multiplying by an arbitrary constant-which need not be the same for the upwelling and the downwelling variants-in order to match the observed magnitudes; we use this demonstration simply to show that such flows are readily available. In Fig. 9 we present one example each of the vertical component [where we plot $W$ from Eqs. (36) and (37)], which exhibit zero-speed boundaries that touch the corresponding boundary of the near-surface flow; this requirement fixes the values of $\Theta_{1}$ and $z_{1}$ in each region. In Fig. 10 we present the associated meridional component (plotted as $\lambda V$ ), computed within the boundaries of the upwelling/downwelling regions.

Finally, we show that a thermocline can be incorporated within flow configuration that we have presented here. Even though our leading-order problem, Eqs. (18)-(21), is based on the solution $\Pi=$ constant, and so we cannot admit regions of different, but constant, density (associated with the jump across a thermocline), we can still introduce a thermocline by virtue of the kinematic condition that must be satisfied as it evolves. If we designate the line of the thermocline at some $\varphi=\varphi_{0}$, for example, at the western end of the Pacific equator, then its path must be described by

$$
W=U \frac{\partial T}{\partial \varphi}+V \frac{\partial T}{\partial \Theta} \quad \text { on } \quad z=T(\varphi, \Theta)
$$

where the thermocline is written as $z=T(\varphi, \Theta)$, with $T\left(\varphi_{0}, \Theta\right)$ given. We assume that the thermocline sits wholly within the region occupied by the EUC core, and then Eq. (38) can be expressed as 


$$
\frac{\partial Q}{\partial \varphi}+\frac{1}{2 \lambda} \frac{\partial}{\partial \Theta}\left\{\left[\frac{-\beta^{\prime}\left(Q^{2}+\lambda^{2} \Theta^{2}\right)+\gamma^{\prime}\left(Q^{2}+\lambda^{2} \Theta^{2}\right)^{2}}{-\beta+2 \gamma\left(Q^{2}+\lambda^{2} \Theta^{2}\right)}\right] \arctan \left(\frac{\lambda \Theta}{Q}\right)\right\}=0,
$$

where $Q=T(\varphi, \Theta)+z_{0}(\varphi)$. Although this equation has a simple, special solution $(T=\Theta A(\varphi)$, representing a tilted thermocline), the restrictions that this imposes on $\beta$ and $\gamma$ imply that this is not relevant to the conditions as observed in the EUC. However, we can use this information as the basis for finding a suitable solution near the equator $(\Theta \rightarrow 0)$. We seek a solution

$$
Q(\varphi, \Theta) \sim Q_{0}(\varphi)+\lambda \Theta Q_{1}(\varphi) \text { as } \Theta \rightarrow 0,
$$

which gives

$$
\gamma Q_{0}^{4}-\beta Q_{0}^{2}=\text { constant } \quad \text { and } \quad Q_{1}=\frac{q}{Q_{0}^{2}\left(\beta-2 \gamma Q_{0}^{2}\right)},
$$

where $q$ is a constant. Here, the former result shows that the speed of the flow at the depth of the thermocline along the line of the equator is constant, as the flow evolves in the azimuthal direction; the latter condition confirms that the thermocline is tilted, north-south, across the line of the equator.

\section{Discussion}

This work has had three main aims, namely, to show that flows of the type observed along the Pacific equator: 1) require the retention of the vertical velocity component (albeit very small, in physical terms), 2) necessitate some evolution (change) in the azimuthal direction, and 3) demonstrate that the three velocity components are inextricably linked nonlinearly. The first two points are readily accomplished (as we have seen) and, indeed, our detailed results confirm the correctness of our contention. The inclusion of the vertical velocity component $w$, within the leading-order system, is no more than a consequence of the shallow-water approximation: the restriction to shallow water (a thin shell) implies, for consistency, that $w$ is correspondingly small but, as we have shown, it cannot be set to zero at leading order.

The role of $w$ is certainly significant but, arguably, the need for some evolution in the azimuthal direction is paramount. This idea was developed in Constantin and Johnson (2017) in a similar, but less general, scenario; this allowed slow variations of an EUC-type flow in the azimuthal direction in the context of the $\beta$-plane approximation. In this new work, we have not resorted to any such geometrical simplification, even though we have limited the detailed discussion to the neighborhood of the equator. [Indeed, this suggests that an area that is worth further exploration is the corresponding asymptotic solution of Eqs. (13)-(17) in the neighborhood of some $\theta=\theta_{0}$, and away from the equator and the poles. Such an analysis will mimic the ideas presented here, but generalize to solutions which do not satisfy the constraint $V \rightarrow 0$ as $\theta \rightarrow \theta_{0}$.]

The development of the nonlinear structure follows quite closely the analysis presented in Constantin and Johnson (2017), but here we can allow a flow $u$ in the azimuthal direction which admits a general profile in the $(\theta, z)$ plane. The introduction of a pseudostreamfunction also follows this earlier pattern, producing $v$ and $w$ components that are proportional to $u$, the proportionality being nonlinear functionals of the $u$ component. This alone confirms the intricate nonlinear, threedimensional nature of the solution. To proceed with the construction of a solution, we first specify the $u$ component of the velocity; this must necessarily evolve in the azimuthal direction, but we do not need to prescribe the details for the purpose of this analysis. Nevertheless, we must make some choices, locally, in order to produce physically relevant solutions that describe the 3D flow field. This important observation has two immediate consequences. First, an issue of some practical significance, and certainly of mathematical interest, is the characterization of the various types of flow that correspond to different choices of the parameters, that is, the evaluations at a given $\varphi$. This is an extensive undertaking, and quite beyond the aims of this initial study. Second, and directly relevant to the description of a solution valid along the equator, is the fact that any solution will necessarily evolve in the azimuthal direction, and so the structure of the 3D flow is likely to change quite dramatically. Precisely this phenomenon was encountered in Constantin and Johnson (2017), with cells appearing and disappearing, for example, as $\varphi$ changed; numerical investigations of this phenomenon have been undertaken by Basu $(2018,2019)$. All this makes clear that the next phase of this project is to examine how a detailed representation of the Pacific EUC, for example, develops from west to east. In the light of the flow patterns described here [and the results reported in Constantin and Johnson (2017)], we can expect some substantial changes to the flow field as $\varphi$ varies along the equator.

The particular solution that we have presented represents a model of the Pacific EUC. The chosen $u$ component comprises an elliptical core, a little below the surface, with a maximum at its center (and zero on its boundary) 
moving eastward. The surface layer is the westward flow, with a structure which reproduces the observational data (see Johnson et al. 2001). This leads directly to expressions for the other two components (defined in the region where $u$ is given), although the production of relevant (i.e., physically realistic) solutions requires a choice to be made for the values of the parameters that represent the $\varphi$ dependence, but at a given station. An example of $v$ and $w$ has been presented; this shows the flow away from the equator near the surface, and closed regions below this, for $v$, and upwelling near the surface around the equator (and otherwise a complicated flow pattern below this) for $w$. Furthermore, we have seen that the thermocline can be included, and that its path-a surface in $3 \mathrm{D}-$ is governed by an equation that incorporates both evolution in the azimuthal direction, and a structure in the meridional direction, although we have yet to find an exact solution that holds within the region covered by the EUC core. Finally, we also showed how solutions can be obtained in the region where $u=0$. As a simple example, we have produced a solution which recovers the observed upwelling and downwelling, away from the EUC. Clearly, many other choices can be made, which might improve the relevance to flows in the equatorial Pacific, or open the way to applying a similar approach to other flows.

In conclusion, this work has shown the importance of retaining the vertical component of the velocity field, and of the overarching need for a flow that varies in the azimuthal direction. The upshot is a fully nonlinear, threedimensional theory that, with careful choice of the relevant parameters, can reproduce much that is observed in these flow fields. In short, the three components are intimately linked: they cannot be arbitrarily assigned for, if one is known (perhaps driven by mechanisms from other parts of the ocean, or induced by wind effects), then the other two are completely determined. Nevertheless, as we have seen, this leaves a lot that needs further attention, particularly a study of the various forms that such flows might take, and the consequences for the evolution of the full, 3D flow field in the azimuthal direction.

Acknowledgments. The support of the WWTF Grant MA16-009 is acknowledged. The authors are grateful for helpful comments from the referees.

\section{APPENDIX}

\section{Velocity Components and Streamfunctions}

The velocity components, obtained from Eqs. (23) and (24), require the construction of $\psi_{z}$ and $\psi_{\Theta}$ from Eq. (30); these are

$$
\begin{gathered}
\psi_{z}=\frac{z+z_{0}}{(-\beta+2 \gamma \zeta)^{2}}\left(\beta \beta^{\prime}-2 \beta \gamma^{\prime} \zeta+2 \gamma \gamma^{\prime} \zeta^{2}\right) \arctan \left(\frac{\lambda \Theta}{z+z_{0}}\right) \\
-\frac{1}{2} \lambda \Theta\left(\frac{-\beta^{\prime}+\gamma^{\prime} \zeta}{-\beta+2 \gamma \zeta}\right), \text { and } \\
\psi_{\Theta}=z_{0}^{\prime}+\frac{\lambda \Theta}{(-\beta+2 \gamma \zeta)^{2}}\left(\beta \beta^{\prime}-2 \beta \gamma^{\prime} \zeta\right. \\
\left.+2 \gamma \gamma^{\prime} \zeta^{2}\right) \arctan \left(\frac{\lambda \Theta}{z+z_{0}}\right) \\
+\frac{1}{2}\left(z+z_{0}\right)\left(\frac{-\beta^{\prime}+\gamma^{\prime} \zeta}{-\beta+2 \gamma \zeta}\right)
\end{gathered}
$$

where $\zeta=\left[z+z_{0}(\varphi)\right]^{2}+\lambda^{2} \Theta^{2}$ and the prime denotes the derivative with respect to $\varphi$. The expression for $\psi(\varphi, \Theta, z)$ in the region $|\Theta|>\Theta_{0}$ is

$$
\begin{aligned}
\psi= & \Theta z_{0}^{\prime}+\frac{1}{2 \lambda} \frac{\alpha^{\prime}-\beta^{\prime} \zeta+\gamma^{\prime} \zeta^{2}}{-\beta+2 \gamma \zeta} \\
& \times\left(\frac{1}{\sqrt{k-1}} \ln \left\{\frac{\lambda\left[(k-1) \Theta \mp k \Theta_{0}\right]+\sqrt{k-1}\left(z+z_{0}\right)}{\sqrt{(k-1)\left(\zeta-\lambda^{2} \Theta^{2}\right)} \mp \lambda \Theta_{0}}\right\}\right. \\
& \left. \pm \arctan \left(\frac{\lambda \Theta_{0}}{\sqrt{\zeta-\lambda^{2} \Theta^{2}}}\right)\right)
\end{aligned}
$$

where the signs are ordered according to $\Theta>\Theta_{0}$, $\Theta<-\Theta_{0}$.

\section{REFERENCES}

Basu, B., 2018: On a three-dimensional nonlinear model of Pacific equatorial ocean dynamics: Velocities and flow paths. Oceanography, 31, 51-58, https://doi.org/10.5670/oceanog.2018.309.

_- 2019: Some numerical investigations into a nonlinear three-dimensional model of the Pacific equatorial ocean flows. Deep-Sea Res. II, 160, 7-15, https://doi.org/10.1016/ j.dsr2.2018.11.013.

Boyd, J. P., 2018: Dynamics of the Equatorial Ocean. Springer, $517 \mathrm{pp}$.

Constantin, A., 2012: An exact solution for equatorially trapped waves. J. Geophys. Res., 117, C05029, https://doi.org/10.1029/ 2012JC007879.

2014: Some nonlinear, equatorially trapped, nonhydrostatic internal geophysical waves. J. Phys. Oceanogr., 44, 781-789, https://doi.org/10.1175/JPO-D-13-0174.1.

—_, and R. S. Johnson, 2016: An exact, steady, purely azimuthal equatorial flow with a free surface. J. Phys. Oceanogr., 46, 1935-1945, https://doi.org/10.1175/JPO-D-15-0205.1.

— , and — 2017: A nonlinear, three-dimensional model for ocean flows, motivated by some observations of the Pacific Equatorial Undercurrent and thermocline. Phys. Fluids, 29, 056604, https://doi.org/10.1063/1.4984001.

__, and —_, 2019: Ekman-type solutions for shallow-water flows on a rotating sphere: A new perspective on a classical 
problem. Phys. Fluids, 31, 021401, https://doi.org/10.1063/ 1.5083088 .

Gill, A., 1982: Atmosphere-Ocean Dynamics. International Geophysics Series, Vol. 30, Academic Press, 662 pp.

Henry, D., 2016: Equatorially trapped nonlinear water waves in a betaplane approximation with centripetal forces. J. Fluid Mech., 804, R1, https://doi.org/10.1017/jfm.2016.544.

,2018: Nonlinear features of equatorial ocean flows. Oceanography, 31, 22-27, https://doi.org/10.5670/oceanog.2018.305.

Johnson, G. C., M. J. McPhaden, and E. Firing, 2001: Equatorial Pacific ocean horizontal velocity, divergence, and upwelling. J. Phys. Oceanogr., 31, 839-849, https://doi.org/10.1175/15200485(2001)031<0839:EPOHVD>2.0.CO;2.
Johnson, R. S., 2018: The value of asymptotic theories in physical oceanography. Oceanography, 31, 14-21, https:// doi.org/10.5670/oceanog.2018.304.

Kessler, W. S., G. C. Johnson, and D. W. Moore, 2003: Sverdrup and nonlinear dynamics of the Pacific equatorial currents. J. Phys. Oceanogr., 33, 994-1008, https://doi.org/10.1175/15200485(2003)033<0994:SANDOT $>2.0$. CO 2 .

Rowe, G. D., E. Firing, and G. C. Johnson, 2000: Pacific equatorial subsurface countercurrent velocity, transport, and potential vorticity. J. Phys. Oceanogr., 30, 1172-1187, https://doi.org/ 10.1175/1520-0485(2000)030<1172:PESCVT>2.0.CO;2.

Talley, L. D., G. L. Pickard, W. J. Emery, and J. H. Swift, 2011: Descriptive Physical Oceanography: An Introduction. Elsevier, 560 pp. 